\title{
Multimodal imaging findings of SAPHO syndrome with no skin lesions: A report of three cases and review of the literature
}

\author{
NA DUAN, XIAO CHEN, YONGKANG LIU, JIANHUA WANG and ZHONGQIU WANG \\ Department of Radiology, The Affiliated Hospital of Nanjing University of \\ Chinese Medicine, Nanjing, Jiangsu 210029, P.R. China \\ Received June 3, 2015; Accepted July 28, 2016
}

DOI: 10.3892/etm.2016.3689

\begin{abstract}
Synovitis, acne, palmoplantar pustulosis, hyperostosis and osteitis syndrome (SAPHO) is a rare syndrome that affects the skin, bones and joints. Diagnosis of SAPHO syndrome is established on clinical appearance and imaging features. The present case report described the imaging features of three cases of SAPHO with sternoclavicular joint arthritis but without skin manifestations using multiple imaging modalities, including computed tomography (CT), magnetic resonance imaging (MRI) and bone scintigraphy. The first case was a 52-year-old male who suffered from progressive sternoclavicular arthritis for 2 years. The second case was a 62-year-old female with arthritis in the larger joints for 5 years, particularly on the right thoracic area. The third case was a 44-year-old male who exhibited a slight bulge accompanied by pain in the upper anterior chest wall for 4 years. All of them lacked cutaneous lesions. CT demonstrated sclerosis and hyperostosis with subchondral erosions in the sternocostoclavicular joints. MRI revealed bone marrow edema that was slightly hypointense on T1-weighted imaging, and hyperintense on T2-weighted imaging. Typical 'bull head' signs were observed in bone scintigraphy images. The present case study demonstrated that SAPHO syndrome should be suspected in patients with multifocal osteitis or arthritis affecting the chest wall that lack skin manifestations. Multimodal imaging modalities in combination are helpful for SAPHO diagnosis.
\end{abstract}

\section{Introduction}

Synovitis, acne, palmoplantar pustulosis, hyperostosis, and osteitis (SAPHO) syndrome was initially described as a disease of aberrant bones, joints and skin lesions in 1987 by

Correspondence to: Professor Zhongqiu Wang, Department of Radiology, The Affiliated Hospital of Nanjing University of Chinese Medicine, 155 Hanzhong Road, Nanjing, Jiangsu 210029, P.R. China E-mail: zhq2001us@163.com

Key words: sign synovitis acne pustulosis hyperostosis osteitis syndrome, imaging findings, 'bull head'
Chamot et al (1). SAPHO syndrome can occur at any age and is characterized by repeated remission and recurrence, and it rarely occurs in individuals aged $>60$ years (2). Although a wide spectrum of clinical hallmarks have been previously described for SAPHO syndrome, the osteoarticular and/or dermatological lesions are the most important manifestations. Most patients present with local inflammatory pain, swelling, and limited movement at the site of the active lesions, particularly at the anterior chest wall (ACW) (3). The prevalence of SAPHO is $<1 / 10000$ (4). However, its actual prevalence may be underestimated due to incorrect diagnosis. High incidence rates of SAPHO syndrome have been reported in the European population (5), whereas only occasional cases are reported in Chinese individuals (6-8). Osteitis and hyperostosis are striking features that can be observed in any involved skeletal segments (9). It has been demonstrated that skin manifestations are detected in $63.5 \%$ of patients (4), whereas at least $15 \%$ adults never experience skin manifestations (10). Therefore, diagnosis of SAPHO syndrome may be difficult in certain cases, particularly if the dermatological manifestations are absent (10). The pathogenesis is poorly understood. However, it has been shown that persistent infection with low-virulence pathogens and the autoimmune process triggered by a bacterial or viral pathogen may be associated with SAPHO syndrome (10).

The present case study described the imaging features of three cases of SAPHO with sternoclavicular joint arthritis but without skin manifestations using multiple imaging modalities, including computed tomography (CT), magnetic resonance imaging (MRI) and bone scintigraphy. This study was approved by the Institutional Review Board of the Affiliated Hospital of Nanjing University of Chinese Medicine (Nanjing, China) with waiver of informed consent.

\section{Case reports}

Case 1. A 52-year-old male patient was admitted to the Affiliated Hospital of Nanjing University of Chinese Medicine due to progressive pain at the ACW in March 2008. Over the past 2 years he had recurrently suffered from sternoclavicular arthritis. Initially, the symptoms included swelling, pain, and muscle stiffness with no apparent causes. He was referred to another hospital, but no diagnosis was made and no treatment was administered. As the swelling did not recover and 

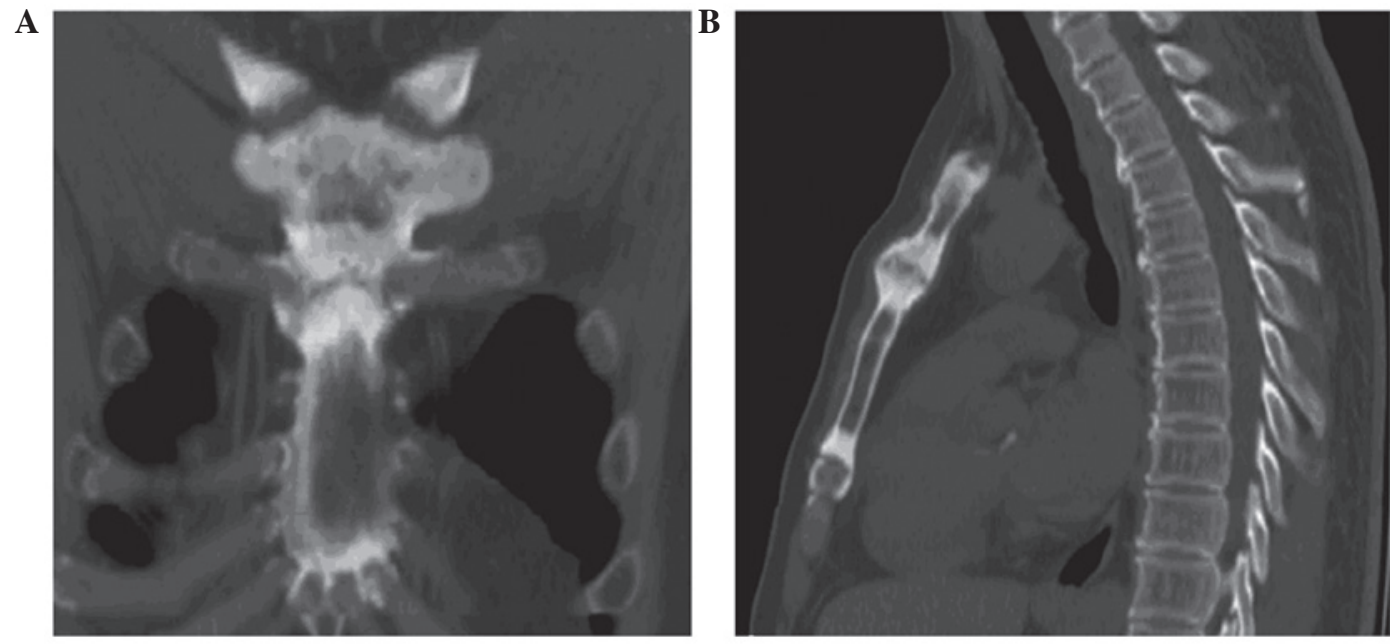

Figure 1. Case 1. (A) Coronal and (B) sagittal reconstructions images of computed tomography in a patient with synovitis, acne, palmoplantar pustulosis, hyperostosis and osteitis syndrome reveal sclerosis and hypertrophy of the manubrium and irregularities and fusion on edges of bilateral manubriosternal, sternoclavicular and sternocostal joints.
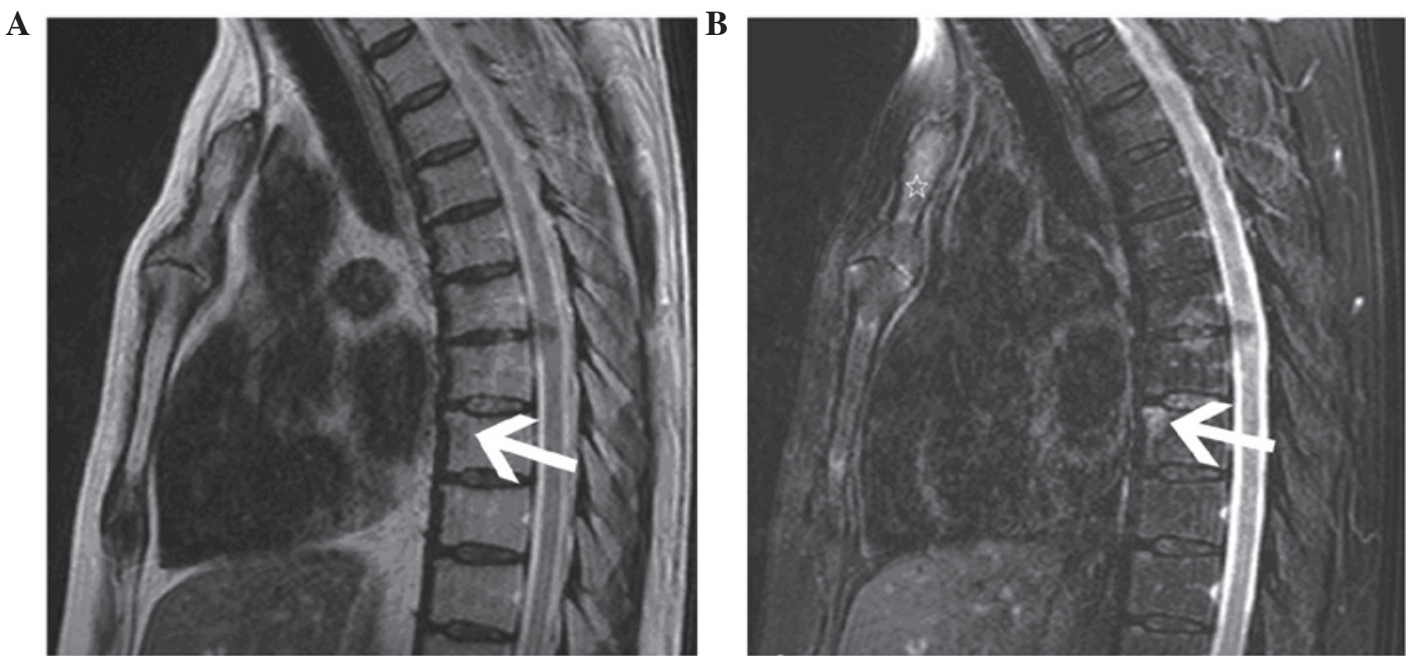

Figure 2. Case 1. Magnetic resonance imaging sagittal (A) T1-weighted image and (B) T2-weighted image with fat saturation show bone marrow edema at the manubriosternal as a sign of arthritis (hollow asterisk). In addition, a bone marrow edema also is observed in the anterior superior border of the eighth thoracic vertebrae (arrow).

became more severe, the patient consulted our institution for further evaluation.

Physical examination indicated swelling and tenderness at the sternoclavicular joint and his body temperature was $37.5^{\circ} \mathrm{C}$. Erythrocyte sedimentation rate (ESR) was $37 \mathrm{~mm} / \mathrm{h}$ (reference range in males, $0-15 \mathrm{~mm} / \mathrm{h}$ ) and C-reactive protein level was $0.83 \mathrm{mg} / \mathrm{dl}$ (reference range, $<1.0 \mathrm{mg} / \mathrm{dl}$ ), which indicated that there was slight inflammatory reaction. Other laboratory data, such as rheumatoid factor and anti-nuclear antibody tests, were negative.

CT scanning demonstrated sclerosis and hyperostosis with subchondral erosions in the sternocostoclavicular joints, the medial end of clavicle, sternum, first rib and costal cartilage (Fig. 1). MRI indicated bone marrow oedema in the manubrium and the eighth thoracic vertebral corner on T1- (Fig. 2A) and T2-weighted (Fig. 2B) imaging. Bone scintigraphy with ${ }^{99 \mathrm{~m}} \mathrm{Tc}-\mathrm{methylene}$ diphosphonate showed an accumulation of tracer in the sternoclavicular and manubri- osternal joints. The typical 'bulls head' sign was observed, and was also detected by helical CT imaging with a volume rendering technique (Fig. 3). Treatment with ibuprofen [100 mg orally, twice daily (bid); Taicheng Pharmaceutical Co., Ltd., Guangdong, China] and prednisone (30 mg orally, once daily; Xinyi Pharmaceutical Co., Ltd., Shanghai, China) was initiated. The patient reached full remission at the end of a 3-month follow-up.

Case 2. A 62-year-old female was admitted to the Affiliated Hospital of Nanjing University of Chinese Medicine with pain in the right thoracic area with no apparent cause in May 2009. She had suffered from pain for 5 years with repeated remission and relapse. Subsequent to her admission, hypesthesia and muscle weakness of upper chest wall on the right side appeared and gradually deteriorated. Pain persisted and was not associated with any noticeable early morning stiffness. The patient did not have a history of skin lesions. Physiotherapy could not relieve her pain; therefore, she 

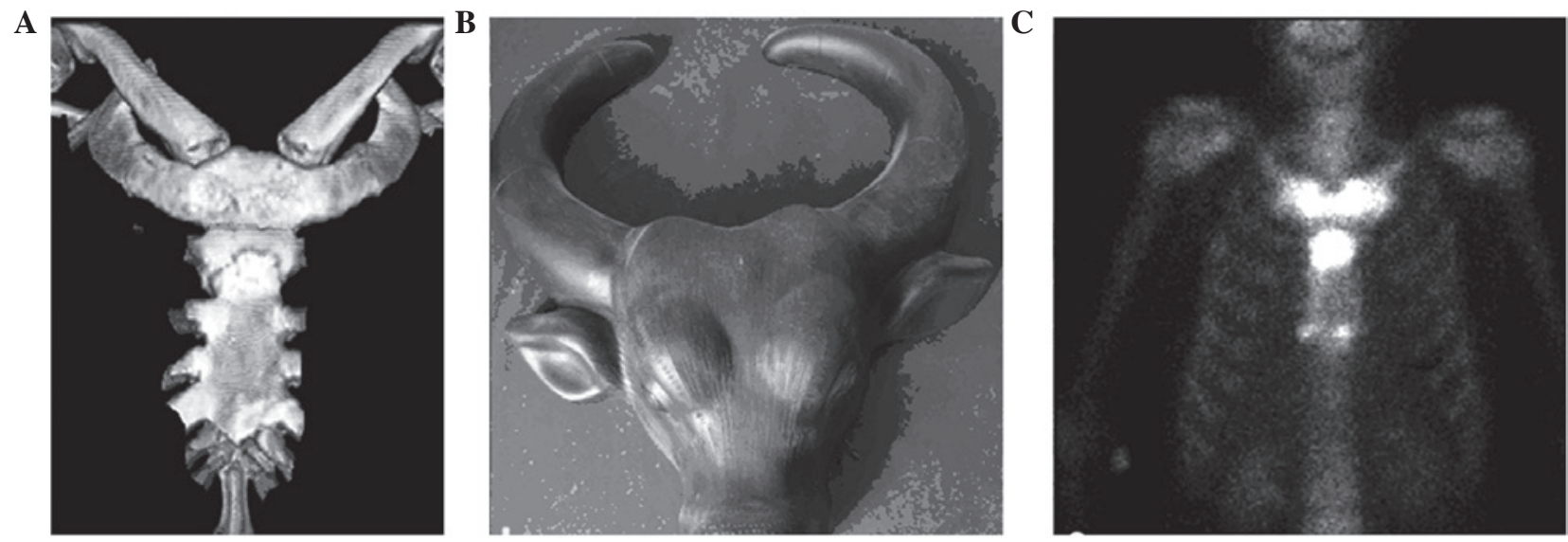

Figure 3. Case 1. Volume rendering of helical computed tomography (A) shows sclerosis and fusion of the sternocostal joints and manubriosternal joints, (B) with a likening to a bull head. (C) Bone scintigraphy with ${ }^{99 m}$ Tc-methylene-diphosphonate complex also indicated the classic 'bull's head' sign due to increased uptake in the manubrium and bilateral sternoclavicular joints.

A

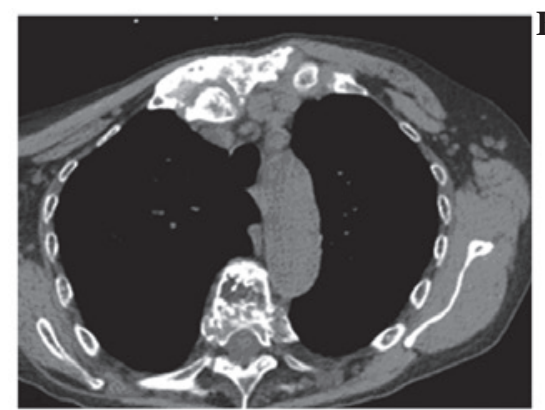

B

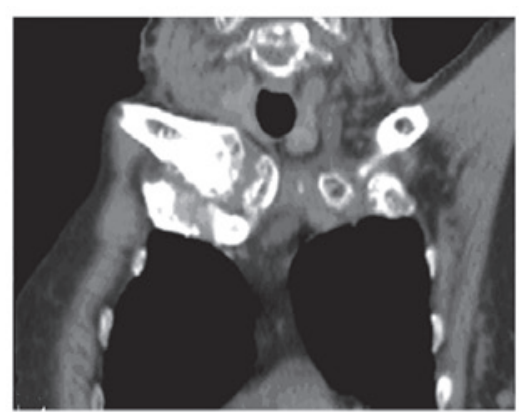

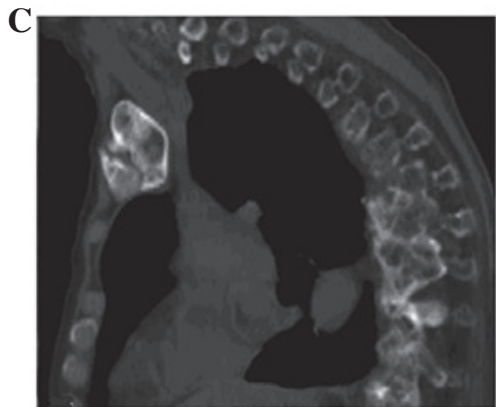

Figure 4. Case 2. (A) Axial (soft tissue window) and (B) coronal reconstructions (bone window) of computed tomography reveal expansion and sclerosis in the right clavicle and first rib. Joint space narrowing and erosive damage at the left sternoclavicular joint (white arrow) were also observed. (C) Sagittal reconstruction (bone window) shows hyperostosis and fusion of the multiple consecutive thoracic vertebrae.

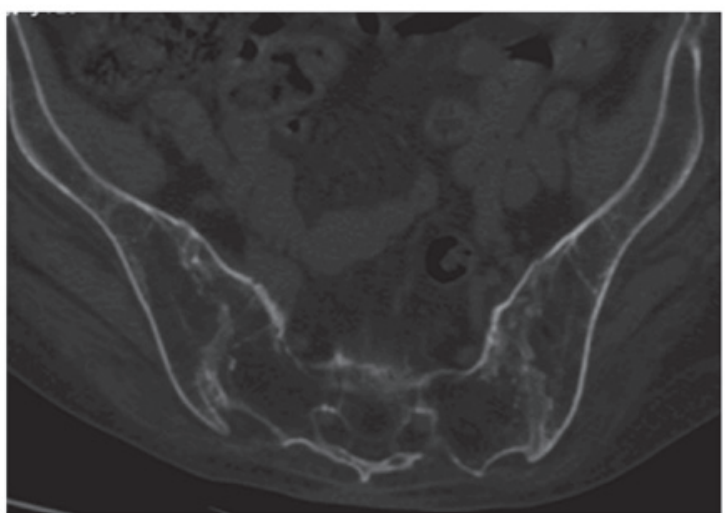

Figure 5. Case 2. Axial (bone window) computed tomography scan shows moderate subchondral sclerosis and cortical erosion at both sacroiliac joints.

underwent therapy for rheumatoid arthritis, but no obvious amelioration was detected. Physical examination indicated that she had no remarkable neuronal responses and tenderness was detected on both knees, her right shoulder, and right sternoclavicular joint with conspicuous swelling.

ESR was $21 \mathrm{~mm} / \mathrm{h}$ (reference range in females, $0-20 \mathrm{~mm} / \mathrm{h}$ ) and C-reactive protein level was $7.22 \mathrm{mg} / \mathrm{dl}$ (reference range, $<1.0 \mathrm{mg} / \mathrm{dl}$ ), which indicated the presence of a slight inflam- matory reaction. Results of routine blood testing were normal. Tumor marker [carcinoembryonic antigen (CEA), CA125 and alkaline phosphatase (ALP)] and tuberculin tests were negative.

CT imaging revealed hyperostosis at the right sternocostoclavicular joints and severe destruction with marginal sclerosis at multiple thoracic vertebras (Fig. 4). Joint space narrowing and erosive damage at the bilateral sacroiliac joints (Fig. 5) was also observed. Whole-body bone scintigraphy with ${ }^{99 \mathrm{~m}} \mathrm{Tc}$-methylene diphosphonate indicated increased tracer uptake in the sternoclavicular joint, upper thoracic vertebra, shoulders, and the knee and ankle joints (Fig. 6). Treatment with ibuprofen (100 bid, orally) and sulfasalazine (500 mg orally, four times daily; Xinyi Pharmaceutical Co., Ltd.).was initiated. The patient reached partial remission at the end of a 3-month follow-up.

Case 3. A 44-year-old male patient was admitted to the Affiliated Hospital of Nanjing University of Chinese Medicine complaining of a slight bulge accompanied by pain at the upper ACW in May 2014. Inadequate attention was paid to the lesion as the patient had no any other systemic complaints with the exception of an indolent mass at his left sternocostoclavicular joints for the past 4 years. The mass had gradually increased in size over the previous year.

Physical examination showed the there was tenderness and swelling at the left ACW. ESR and C-reactive protein 
A

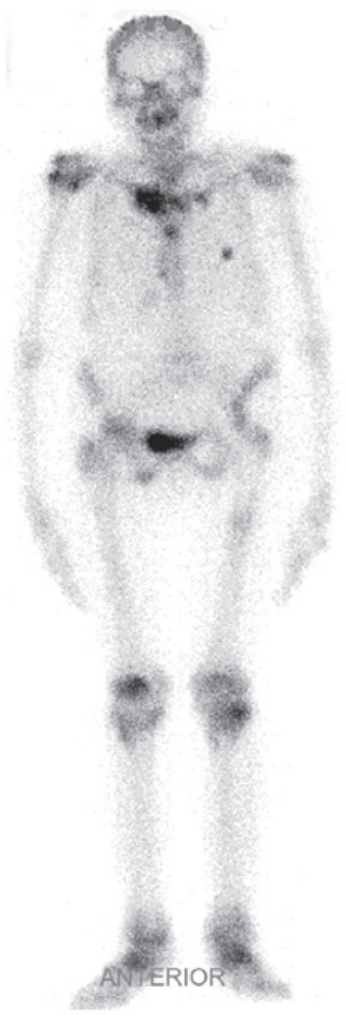

B

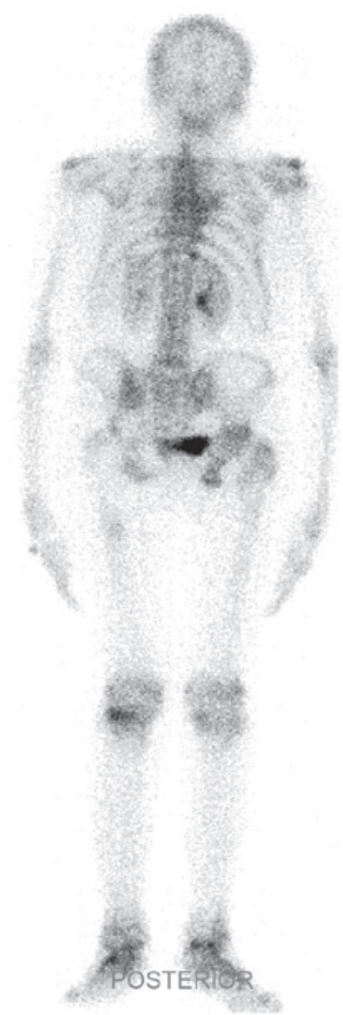

Figure 6. Case 2. (A) Anterior and (B) posterior view of whole-body bone scintigraphy with ${ }^{99 \mathrm{~m}} \mathrm{Tc}$-methylene-diphosphonate. Increased tracer uptake was observed in both sternoclavicular joints (predominantly in the right), the sternum, the right parts of the upper thoracic vertebrae, the first ribs and the middle of the left seventh anterior rib. Increased uptake was also observed in the shoulders, and the knee and bilateral ankle joints, which was considered to be indicative of osteoarthritis.

level were within the normal ranges. Human leukocyte antigen (HLA) B27 antigen was positive. Tumor marker (CEA, CA125, prostate-specific antigen and ALP) and tuberculin test results were normal.

CT scanning revealed subarticular erosions and regional sclerosis at the sternoclavicular joint (Fig. 7). Bone marrow edema at the right collarbone was detected by MRI axial T2-weighted imaging and T2-weighted imaging with fat saturation (Fig. 8). Although no skin lesions developed during the clinical course, the diagnosis of SAPHO syndrome was made based on the presence of osteoarthritis at the sternoclavicular joint. Ibuprofen (200 mg orally, twice daily; Xiuzheng Pharmaceutical Co., Ltd., Jilin, China) and methotrexate (7.5 mg orally, once weekly; Xinyi Pharmaceutical Co., Ltd.) were administered. The patient reached partial remission at the end of a 2-month follow-up.

\section{Discussion}

SAPHO syndrome is a well-documented rare chronic disorder, which is characterized by abnormal osteoarticular manifestations and a range of chronic dermatosis (11). The majority of published papers in this field are case reports or small series case studies. The natural history and long-term evolution of the disease have not been investigated extensively. Among patients with SAPHO syndrome, $65-90 \%$ presented with damage to the anterior chest (9). Involvement of multiple ACW sites such as the sternoclavicular, manubriosternal, costochondral, and costosternal junctions should be considered as an important criterion for diagnosis (10).
Diagnosis of SAPHO syndrome is based on the presence of at least one of four diagnostic criteria (12): i) Osteoarticular manifestations with severe acne; ii) osteoarticular manifestations with palmoplantar pustulosis; iii) hyperostosis involving either the ACW, spine, or limbs, with or without dermatosis; and iv) recurrent multifocal chronic osteomyelitis involving the axial or peripheral skeleton, with or without dermatosis. Skin symptoms. including palmoplantar pustulosis or/and severe acne, can be helpful for diagnosing SAPHO syndrome. In addition, other dermatoses associated with SAPHO include pustular psoriasis, hidradenitis suppurativa, and psoriasis vulgaris (13). Some researchers have reported that skin lesions were not observed in $16 \%$ of patients, and the osteoarticular manifestation antedates the skin manifestation in $32 \%$ of patients $(2,14,15)$. Skeletal symptoms and skin disease do not always occur in parallel (8), which has an influence on the diagnosis. These findings indicate that skin lesions are not a necessary criteria for SAPHO diagnosis if patients exhibit hyperostosis or multifocal chronic osteomyelitis. Osteoarticular manifestations include hyperostosis, osteitis, synovitis and arthropathy. Osteitis and hyperostosis are striking features that can be observed in any involved skeletal segments (9). The main affected site is the ACW, but other bones may also be involved (9). Osteitis or hyperostosis was observed in the three cases described in the present case report. Therefore, the three cases in the present report were diagnosed with SAPHO syndrome with specific osteoarthritic manifestation at certain sites in the absence of lesions. 
A

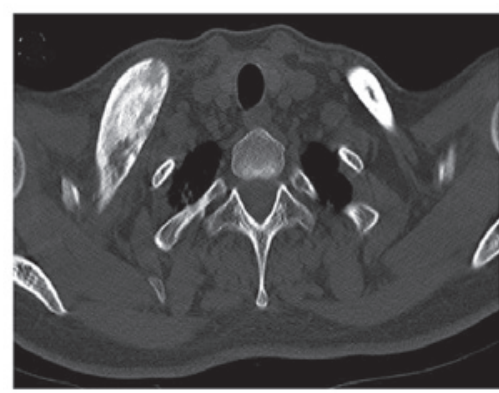

B

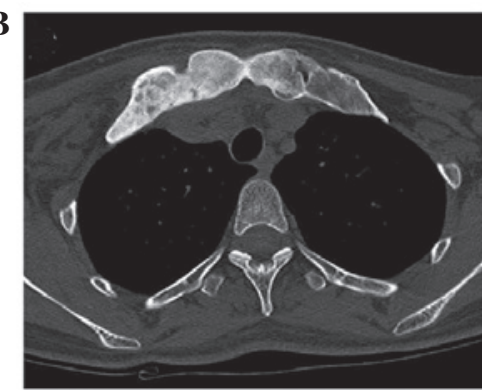

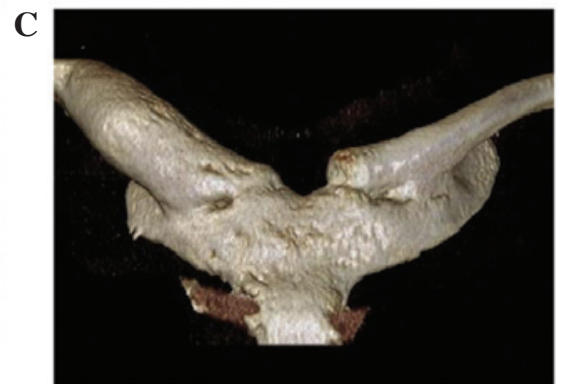

Figure 7. Case 3. (A and B) Axial computed tomography (soft tissue window) and (C) volume rendering reconstruction images indicating hypertrophy and fusion on the edges of bilateral sternoclavicular joints.
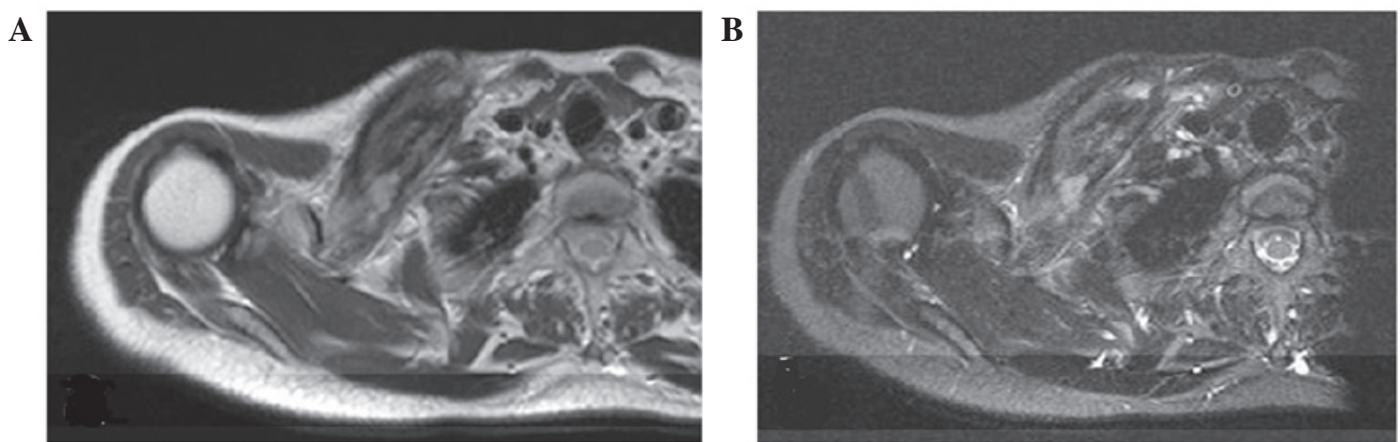

Figure 8. Case 3. (A) Axial T2-weighted and (B) T2-weighted with fat saturation images of the right chest demonstrated bone marrow edema at the right collarbone.

The cause and pathogenesis of SAPHO syndrome remains poorly understood (15). As such, SAPHO syndrome can be misdiagnosed as a tumor or infectious disease (16). Misdiagnosed benign tumors include chronic infectious osteomyelitis, Paget's disease, spondylarthropathies and osteitis condensans of the clavicle. Malignant entities that should be considered are multiple myeloma, extramedullary plasmacytoma, osteosarcoma and bone metastasis. When radiological findings are confused with these entities, a history of skin lesions, results of other laboratory tests and examination with multiple imaging modalities may be helpful for differential diagnosis. Radiologists should recommend bone scintigraphy or positron emission tomography-CT to reveal asymptomatic manifestations. Standard uptake values can be used to differentiate SAPHO syndrome from malignancies, infections processes and metastatic diseases $(17,18)$.

There is no specific biomarker for SAPHO syndrome. ESR and C-reaction protein levels can be moderately elevated, and it has been shown that HLA-B27 is present in $15-33 \%$ (1) or $4-14 \%$ (9) of patients. In the present study, elevation of ESR and $\mathrm{C}$-reaction were detected in two cases (cases 1 and 2) and HLA-B27 was present in one case (case 3).

SAPHO syndrome remains difficult to diagnose. Before a correct diagnosis is made, patients are often subjected to multiple imaging examinations and unsuccessful therapy. Radiologists have a key role in the early diagnosis for SAPHO syndrome $(19,20)$. Radiographs, bone scintigraphy, CT and MRI are invaluable diagnostic tools. As a more sensitive imaging modality, CT has the ability to detect abnormalities in the sternoclavicular area that are not obvious on radiographs. Bone and soft-tissue edemas are well recognized on MRI, particularly on short-tau inversion recovery sequences, which can be useful in differentiating between active and inactive lesions (21). MRI is radiation-free and is well suited for patients who require repeated follow-up examinations. Whole-body bone scintigraphy is a useful investigation technique in the detection of relatively inactive and subclinical lesions (22). Predominant high tracer uptake in the sternoclavicular region appears to mimic a 'bull head', with the manubrium representing the upper skull of the bull and the horns corresponding to the inflamed sternoclavicular joints and the adjacent claviculae $(23,24)$. In the present study, sclerosis or hyperostosis with subchondral erosions in the sternocostoclavicular joints or manubrium were observed on CT images. MRI images also showed bone marrow oedema in case 2 . Whole-body bone scintigraphy indicated multiple lesions, including lesions in the sternoclavicular joint, upper thoracic vertebra, the shoulders, the knee and ankle joints, and the typical 'bull's head' sign in case 2. These findings suggest that multiple modalities imaging in combination may be helpful for the diagnosis of SAPHO syndrome.

$\mathrm{ACW}$ and sternocostoclavicular joints were the main regions affected in the present cases. It has been shown that the lesions of SAPHO syndrome may be age dependent (25). In children, SAPHO syndrome often affects long bones, followed by the clavicle and spine (15). In adults, sternocostoclavicular joints and ACW are predominately affected, followed by spine (19). Sweeney et al (13) indicated that ribs may also be involved. Laredo et al (18) showed that vertebral corner erosion was a consistent MR imaging finding of 
SAPHO. Involvement of spine and ribs were also found in our cases (spine, cases 1 and 2; ribs, cases 2 and 3). Additional regions were affected in case 2 , as detected by whole-body bone scintigraphy, which suggested that whole-body bone scintigraphy may have a relevant role in the detection of lesion localizations.

In conclusion, SAPHO syndrome is characterized by progressive hyperostosis and the eventual destruction of sternoclavicular joints. Multiple bones may also be involved, including the spine and ribs. Diagnosis remains challenging in the absence of skin lesions and multiple imaging modalities may aid the early and correct diagnosis of SAPHO syndrome.

\section{References}

1. Chamot AM, Benhamou CL, Kahn MF, Beraneck L, Kaplan G and Prost A: Acne-pustulosishyperostosis-osteitis syndrome: Results of a national survey. 85 cases. Rev Rhum Mal Osteoartic 54: 187-196, 1987 (In French).

2. Carneiro S and Sampaio-Barros PD: SAPHO syndrome. Rheum Dis Clin North Am 39: 401-418, 2013.

3. Judex AG, Freyschmidt J, Feuerbach S, Schölmerich J and Müller-Ladner U: Sequential combination therapy leading to sustained remission in a patient with SAPHO syndrome. Open Rheumatol J 3: 18-21, 2009.

4. Govoni M, Colina M, Massara A and Trotta F: SAPHO syndrome and infections. Autoimmun Rev 8: 256-259, 2009.

5. Sallés M, Olivé A, Perez-Andres R, Holgado S, Mateo L, Riera E and Tena X: The SAPHO syndrome: A clinical and imaging study. Clin Rheumatol 30: 245-249, 2011.

6. Zhang LL, Zhao JX and Liu XY: Successful treatment of SAPHO syndrome with severe spinal disorder using entercept: A case study. Rheumatol Int 32: 1963-1965, 2012.

7. Zhao Z, Li Y, Li Y, Zhao H and Li H: Synovitis, acne, pustulosis, hyperostosis and osteitis (SAPHO) syndrome with review of the relevant published work. J Dermatol 38: 155-159, 2011.

8. Song X, Sun W, Meng Z, Gong L, Tan J, Jia Q, Yu C and Yu T: Diagnosis and treatment of SAPHO syndrome: A case report. Exp Ther Med 8: 419-422, 2014.

9. Colina M, Govoni M, Orzincolo C and Trotta F: Clinical and radiologic evolution of synovitis, acne, pustulosis, hyperostosis, and osteitis syndrome: A single center study of a cohort of 71 subjects. Arthritis Rheum 61: 813-821, 2009.

10. Nguyen MT, Borchers A, Selmi C, Naguwa SM, Cheema G and Gershwin ME: The SAPHO syndrome. Semin Arthritis Rheum 42: 254-265, 2012.
11. Takigawa T, Tanaka M, Nakahara S, Sugimoto Y and Ozaki T: SAPHO syndrome with rapidly progressing destructive spondylitis: Two cases treated surgically. Eur Spine J 17 (Suppl 2): 331-337, 2008

12. Benhamou CL, Chamot AM and Kahn MF: Synovitis-acnepustulosis hyperostosis-osteomyelitis syndrome (SAPHO). A new syndrome among the spondyloarthropathies? Clin Exp Rheumatol 6: 109-112, 1988

13. Sweeney SA, Kumar VA, Tayar J, Weber DM, Safdar A, Alonso C and Hymes S: Case 181: Synovitis acne pustulosis hyperostosis osteitis (SAPHO) syndrome. Radiology 263: 613-617, 2012.

14. Depasquale R, Kumar N, Lalam RK, Tins BJ, Tyrrell PN, Singh J and Cassar-Pullicino VN: SAPHO: What radiologists should know. Clin Radiol 67: 195-206, 2012.

15. Earwaker JW and Cotten A: SAPHO: Syndrome or concept? Imaging findings. Skeletal Radiol 32: 311-327, 2003.

16. Freyschmidt J and Sternberg A: The bullhead sign: Scintigraphic pattern of sternocostoclavicular hyperostosis and pustulotic arthroosteitis. Eur Radiol 8: 807-812, 1998.

17. Fritz P, Baldauf G, Wilke HJ and Reitter I: Sternocostoclavicular hyperostosis: Its progression and radiological features. A study of 12 cases. Ann Rheum Dis 51: 658-664, 1992.

18. Laredo JD, Vuillemin-Bodaghi V, Boutry N, Cotten A and Parlier-Cuau C: SAPHO syndrome: MR appearance of vertebral involvement. Radiology 242: 825-831, 2007.

19. Van Doornum S, Barraclough D, McColl G and Wicks I: SAPHO: Rare or just not recognized? Semin Arthritis Rheum 30: 70-77, 2000

20. Pahlavan PS and Leslie WD: Multiple imaging findings in SAPHO syndrome. Clin Nucl Med 33: 912-915, 2008.

21. Patel CN, Smith JT, Rankine JJ and Scarsbrook AF: F-18 FDG PET/CT can help differentiate SAPHO syndrome from suspected metastatic bone disease. Clin Nucl Med 34: 254-257, 2009.

22. Boutin RD and Resnick D: The SAPHO syndrome: An evolving concept for unifying several idiopathic disorders of bone and skin. AJR Am J Roentgenol 170: 585-591, 1998.

23. Zemann W, Pau M, Feichtinger M, Ferra-Matschy B and Kaercher H: SAPHO syndrome with affection of the mandible: Diagnosis, treatment, and review of literature. Oral Surg Oral Med Oral Pathol Oral Radiol Endod 111: 190-195, 2011.

24. Shikino K, Ikusaka M and Hirota Y: Magnetic resonance imaging of sternoclavicular joint arthritis due to SAPHO syndrome. Int J Case Rep Images 5: 462-464, 2014.

25. Quirico Rodríguez M, Casáns Tormo I, Redal Peña MC and López Castillo V: The importance of bone scintigraphy in the diagnosis of SAPHO syndrome. Rev Esp Med Nucl 29: 127-130, 2010 (In Spanish). 